\title{
NRF-1 directly regulates TFE3 and promotes the proliferation of renal cancer cells
}

\author{
WENYUAN ZHUANG $^{1 *}$, XIANG DONG ${ }^{2 *}$, BO WANG $^{1}$, NING LIU ${ }^{1}$, \\ HONGQIAN GUO $^{1}$, CHUNNI ZHANG ${ }^{3}$ and WEIDONG GAN ${ }^{1}$ \\ ${ }^{1}$ Department of Urology, Nanjing Drum Tower Hospital, Medical School of Nanjing University; ${ }^{2}$ Department of Urology, \\ Drum Tower Clinical Medical School of Nanjing Medical University; ${ }^{3}$ Department of Clinical Laboratory, \\ Jinling Hospital, Nanjing University School of Medicine, Nanjing University, Nanjing, Jiangsu 210008, P.R. China
}

Received November 27, 2020; Accepted June 7, 2021

DOI: $10.3892 / \mathrm{ol} .2021 .12940$

\begin{abstract}
The role of transcription factor binding to IGHM enhancer 3 (TFE3) in renal cell carcinoma (RCC) is not well understood. Nuclear respiratory factor $1(\mathrm{NRF}-1)$ may be the positive upstream regulatory gene of TFE3. The aim of the present study was to determine whether NRF-1 could directly regulate the expression of TFE3 and regulate tumorigenesis and progression of RCC through TFE3. Short hairpin RNA (shRNA) was used to silence the expression of NRF-1 in the 786-O human kidney adenocarcinoma cell line and the 293T human embryonic kidney cell line. Luciferase reporter assays were used to determine the relationship between NRF-1 and TFE3. The CHIP experiment was used to verify the actual binding of NRF-1 and TFE3 promoter regions. MitoTimer staining was used to measure mitochondrial biosynthesis. Flow cytometry was used to detect cell cycle and apoptosis. The 786-O and 293T cells were used to examine the underlying mechanism of action. The results demonstrated that NRF-1 could bind to the promoter region of the TFE3 gene and directly regulate the expression of TFE3. Following NRF-1 knockdown, the protein levels of phosphorylated (p)-AKT and p-S6 of mTOR pathway was inhibited, cell cycle progression was blocked, the levels of apoptosis increased, and mitochondrial generation was reduced. Following overexpression of TFE3, the levels of mTOR-associated markers were
\end{abstract}

Correspondence to: Dr Weidong Gan, Department of Urology, Nanjing Drum Tower Hospital, Medical School of Nanjing University, Nanjing University, 321 Zhongshan Road, Nanjing, Jiangsu 210008, P.R. China

E-mail: gwd@nju.edu.cn

Dr Chunni Zhang, Department of Clinical Laboratory, Jinling Hospital, Nanjing University School of Medicine, Nanjing University, 305 Zhongshan Road, Nanjing, Jiangsu 210008, P.R. China

E-mail: zchunni27@hotmail.com

*Contributed equally

Key words: renal cell carcinoma, TFE3, NRF-1, metabolism restored in NRF-1 knockdown cells. These findings suggest that NRF-1 may regulate the mTOR pathway through TFE3 and regulate the energy metabolism, proliferation and growth of cancer cells by directly regulating the expression of TFE3.

\section{Introduction}

MiT/TFE transcriptional factors encodes four distinct genes: MITF, TFEB, transcription factor binding to IGHM enhancer 3 (TFE3) and TFEC (1-5). Structurally, MiT/TFE genes encode a protein with a double-helix leucine zipper motif, a transactivating zone and a domain responsible for DNA contact and binding (1-5). The TFE3 gene is 14,749-bp long and is located on chromosome Xp11.22 (6). The functional domain of the TFE3 gene can fuse with the promoter region of other genes, usually housekeeping genes, resulting in the constitutive overexpression of the TFE3 protein and a condition referred to as Xp11.2-translocation renal cell carcinoma (tRCC) $(7,8)$. The TFE3 protein plays an important role in the development of Xp11.2 tRCC, as well as other types of renal cell carcinoma $(9,10)$. Indeed, high levels of TFE3 protein tend to indicate poor prognosis and fast progression (8). Previous studies have identified that TFE3 binds to coordinated lysosomal expression and regulation sequence elements to induce lysosomal biogenesis and autophagy $(11,12)$. TFE3 also plays a role in cellular energy metabolism. For instance, TFE3 governs insulin signaling and glucose metabolism by upregulating insulin receptor 1 substrate (IRS)-2 and hexokinase enzymes, thus inhibiting lipogenesis and increasing glycogen synthesis $(13,14)$. In addition, TFE3 may promote oncogenesis by regulating the cell cycle in Xp11.2 tRCC $(15,16)$.

Nuclear respiratory factor-1 (NRF-1) is a transcription factor that functions primarily as a positive regulator of genes involved in mitochondrial biogenesis and oxidative phosphorylation $(17,18)$. NRF-1 belongs to the NRF family and, together with NRF-2, participates in the regulation of the expression of components related to respiratory chain subunits, mitochondrial replication and transcription (16). NRF-1 plays a vital role in maintaining mitochondrial oxidative respiration and energy production, as well as regulating cell proliferation and growth (19). In addition, a previous study demonstrated that NRF-1, the main regulator of various 
genes in the nervous system, is associated with neurodegenerative diseases (20). A previous study identified 2,470 NRF-1 target genes in SK-N-SH human neuroblastoma cells using chromatin immunoprecipitation sequencing (ChIP-Seq) (18). The molecular pathways of these genes involve regulation of RNA metabolism, splicing, cell cycle, DNA damage repair, protein translation initiation and ubiquitin-mediated protein degradation, along with mitochondrial respiratory function. TFE3 is also a target gene of NRF-1 (21).

It is well known that tumorigenesis results in a parallel change in cellular metabolism, which is closely related to the biosynthesis of mitochondria and linked to NRF-1 $(22,23)$. Moreover, TFE3 itself is involved in cellular energy metabolism. Previous reports indicated that TFE3 may be one of the downstream target genes of NRF-1 $(1,8,19,22)$. Therefore, the aim of the present study was to determine whether NRF-1 had a direct regulatory effect on TFE3 and whether it could mediate the occurrence and development of tumors through TFE3.

\section{Materials and methods}

Cell culture. The cell lines used in this study included the 786-O human kidney adenocarcinoma cell line (CRL-1932 $\left.{ }^{\mathrm{TM}}\right)$ and the 293T human embryonic kidney cell line (CRL11268 ${ }^{\mathrm{TM}}$ ), which were obtained from the American Type Culture Collection. DMEM supplemented with 10\% FBS (both from Gibco; Thermo Fisher Scientific, Inc.) and $1 \%$ penicillin/streptomycin (Invitrogen; Thermo Fisher Scientific, Inc.) was used to culture cells in a $5 \% \mathrm{CO}_{2}$ humidified incubator at $37^{\circ} \mathrm{C}$.

Gene silencing and plasmid constructs. In the present study, lentiviral vectors were used, and the virus assembly and sequence design were completed by OBiO Technology (Shanghai) Corp. The selected interference vector was pLKD-CMV-Puro-U6-shRNA vector [OBiO Technology (Shanghai) Corp.]. The ccdB toxic gene downstream of the U6 promoter was cut with AgeI and EcoR and the shRNA sequence to be constructed was inserted. Lentiviruses were produced by the transfection of 293T cells [OBiO Technology (Shanghai) Corp.] with Gag/Pal along with VSV-G [OBiO Technology (Shanghai) Corp.]. Cells at 50-60\% confluence were transduced with lentivirus carrying short hairpin RNA (shRNA) targeting the human TFE3 or NRF1 genes [multiplicity of infection, 3; virus titer, $1 \times 10^{8}$ transduction units/ml (TU)]. The transfection reagent was HitransG (cat. no. GCD0252780; Shanghai Genechem Co., Ltd.). The sequences used were as follows: i) NRF1-shRNA, 5'-GTAAGTACAAGAGCATGA T-3'; ii) TFE3-shRNA, 5'-GCTCCGAATTCAGGAACTA-3'; and iii) negative control (NC) shRNA, 5'-TTCTCCGAACGT GTCACGT-3'. The samples were incubated at $37^{\circ} \mathrm{C}$ for $12-16 \mathrm{~h}$, the medium was changed to complete medium and then incubation was continued. After 48-h incubation in a $5 \% \mathrm{CO}_{2}$ humidified incubator at $37^{\circ} \mathrm{C}$. The infected cells were selected using $2 \mu \mathrm{g} / \mathrm{ml}$ of puromycin (cat. no. P8833; Sigma-Aldrich; Merck KGaA) for stable clones.

For overexpression, the cDNA encoding TFE3 was subcloned into pcDNA3.1 (V790-20; Invitrogen; Thermo Fisher Scientific Inc.) with ClonExpress II One Step Cloning kit (cat. no. C112; Vazyme Biotech Co., Ltd.). The primers used for constructing the TFE3 overexpression plasmid were as follows: forward, 5'GCCACCATGTCTCATGCGGCC GAA-3'; reverse, 5'-CAGGACTCCTCTTCCATGCTG-3'. Cells in the logarithmic growth phase (density, 40-50\%) were plated in a 6 -well plate with $3.5 \mu \mathrm{g}$ plasmid per well. The plasmid was transfected using Lipofectamine ${ }^{\circledR} 2000$ (Invitrogen; Thermo Fisher Scientific, Inc.) following the manufacturer's instructions. The plasmid suspension and Lipofectamine ${ }^{\circledR} 2000$ suspension were mixed gently and allowed to stand at room temperature for $20 \mathrm{~min}$ followed by incubation in a $5 \% \mathrm{CO}_{2}$ humidified incubator at $37^{\circ} \mathrm{C}$ for $48 \mathrm{~h}$. The expression plasmids for NRF-1 were constructed according to a previous report (24).

ChIP. ChIP was performed according to the protocol from the Pierce $^{\mathrm{TM}}$ Agarose ChIP kit (Thermo Fisher Scientific, Inc.; cat. no. 26156). Briefly, 293 T cells were fixed with in $1 \%$ formaldehyde in culture medium at room temperature for $5 \mathrm{~min}$. The remaining unreacted formaldehyde was quenched with glycine. Cells were lysed in Buffer 1, centrifuged at 9,000 x g for $3 \mathrm{~min}$ and the supernatant was removed. Pellets were resuspended in Buffer 1, homogenized on ice, then pelleted again. Next, the pellet was resuspended in Buffer 2. The chromatin fraction was sheared by sonication in $1.5 \mathrm{ml}$ siliconized microcentrifuge tubes. The sheared chromatin was immunoprecipitated with either anti-NRF-1 antibody (10 $\mu \mathrm{g} / \mathrm{sample}$; cat. no. ab175932; Abcam) or normal rabbit IgG (1-2 $\mu 1$; cat. no. 26156; Thermo Fisher Scientific Inc.) overnight at $4^{\circ} \mathrm{C}$ with constant rotation. The isolated complexes were washed with IP Wash Buffer 2 and IP Wash Buffer 3 prior to elution. The DNA fragments were then separated from complexes and recovered through column purification (Thermo Fisher Scientific, Inc.; cat. no. 26156). The co-precipitated DNA fragments were identified by quantitative PCR (qPCR) as detailed below using primers. Specific for the TFE3 promoter region: forward primer, 5'-GGTCGTCCGGGGTTAGGTT-3'; reverse primer, 5'-TCCGCTAAGCCATGGAGCTA-3'.

Luciferase reporter assay. The promoter region online analysis software JASPER (http://jaspar.genereg.net/cgi-bin/jaspar_ db.pl) was used to analyze NRF-1 binding sites in the promoter region of TFE3. The TFE3 promoter was obtained from the NCBI and cloned into the pGL4.10 vector (Shanghai Obio Technology Co., Ltd.). The amplified plasmid was verified by restriction enzyme digestion and the monoclonal was in accordance with the theoretical design (Fig. 1C). The sequences of the plasmids were verified using sequencing. The plasmid was transfected using Lipofectamine ${ }^{\circledR} 2000$ (Invitrogen; Thermo Fisher Scientific, Inc.) following the manufacturer's recommendations. Plasmid constructs were co-transfected with pGMLR-TK Renilla luciferase-containing plasmid (cat. no. 11558ES03; Shanghai Yeason Biotechnology Co., Ltd.) as an internal control. Reporter activity was detected using Dual Luciferase Reporter Gene Assay Kit (Beyotime Institute of Biotechnology) according to the manufacturer's instructions. The TFE3 luciferase reporter gene plasmid was transfected into $293 \mathrm{~T}$ cells, interfered with NRF1-shRNA and luciferase activity was detected $48 \mathrm{~h}$ later. The cells were then lysed, and luciferase activity was detected using the Dual-Glo luciferase assay system. Renilla luciferase was used the internal control, the RLU value was obtained by the firefly 
luciferase measurement divided by the RLU value obtained by the Renilla luciferase measurement. According to the obtained ratio, the activation degree of the target reporter gene was assessed among different samples. The sequences of shRNA used were as follows: NRF1-shRNA, 5'-GTAAGTACAAGA GCATGAT-3'; and negative control (NC) shRNA, 5'-TTCTCC GAACGTGTCACGT-3'. The virus assembly and sequence design were completed by Dharmacon.

Western blot analysis. Total cellular proteins were treated with extraction buffer $(50 \mathrm{mM}$ Tris- $\mathrm{HCl}, \mathrm{pH} 7.4 ; 150 \mathrm{mM} \mathrm{NaCl}$; 1\% NP-40 Lysis Buffer; 0.1\% SDS; 1X protease inhibitor cocktail). Whole cell lysates were centrifuged at $12,000 \mathrm{x} \mathrm{g}$ at $4{ }^{\circ} \mathrm{C}$. Total protein was quantified via the BCA assay. Total cellular protein (40 $\mu \mathrm{g} / \mathrm{lane}$ ) was subjected to $10 \%$ SDS-PAGE, then transferred to a PVDF membrane (Roche Diagnostics). Blots were blocked with 5\% non-fat milk in TBS $+0.05 \%$ Tween-20 (Sigma-Aldrich; MerckKGaA) (TBST). The membranes were incubated with primary antibodies overnight at $4^{\circ} \mathrm{C}$. After washing with TBST, HRP-conjugated secondary antibodies were applied at room temperature for $2 \mathrm{~h}$. After antibody incubation, the blots were washed with TBST 5 times for 5 min each time. The protein bands were visualized using an enhanced chemiluminescence detection kit (cat. no. P0018A; Beyotime Institute of Biotechnology) and recorded on a radiographic film (Alpha Innotech Corporation). The following primary antibodies were: Anti-TFE3 (1:2,000; cat. no. HPA023881; Sigma-Aldrich; Merck KGaA); anti-NRF-1 (1:2,000; cat. no. ab175932; Abcam); anti-mTOR (1:5,000; cat. no. ab134903; Abcam); anti-S6 (1:1,000; cat. no. CST 54D2; Cell Signaling Technology Inc.); anti-p-S6 (1:2,000; cat. no. CST D57.2.2E; Cell Signaling Technology Inc.); anti-AKT (1:1,000; cat. no. CST 9272; Cell Signaling Technology Inc.); anti-p-AKT (1:2,000; cat. no. CST 4060; Cell Signaling Technology Inc.); and anti-GAPDH (1:2,500; cat. no. BM3876; Wuhan Boster Biological Technology, Ltd.). The following secondary antibodies were used: anti-Rabbit IgG (1:5,000; BA1054; Wuhan Boster Biological Technology, Ltd.) and anti-Mouse IgG (1:5,000; cat. no. BA1051; Wuhan Boster Biological Technology, Ltd.). Semi-quantitative analysis was performed using ImageJ v.1.8.0 software (National Institutes of Health).

$R N A$ isolation and reverse transcription-quantitative $P C R$ (RT-qPCR). Total RNA was extracted from the 786-O and 293T cells at room temperature using Trizol ${ }^{\circledR}$ reagent (Invitrogen; Thermo Fisher Scientific, Inc.) and reverse-transcribed using HiScript Q RT Supermix for qPCR (Vazyme Biotech Co., Ltd., cat. no. R122-01) according to the manufacturer's instructions. In RT process, reaction system including RNA was incubated at $42^{\circ} \mathrm{C}$ for $60 \mathrm{~min}$ to synthesize cDNA, then incubated at $80^{\circ} \mathrm{C}$ for $10 \mathrm{~min}$ to inactivate the reverse transcriptase and terminate the RT reaction. For qPCR analysis, SYBR Green ER (Roche Diagnostics) was used according to the manufacturer's protocol. Total RNA samples were prepared from control and various treated cells and analyzed by real-time PCR analysis using a 7300 system (Applied Biosystems; Thermo Fisher Scientific, Inc.). The thermocycling conditions were as follows: amplification was done for 25 cycles, each with denaturation at $95^{\circ} \mathrm{C}$ for $30 \mathrm{sec}$, annealing at $60^{\circ} \mathrm{C}$ for $30 \mathrm{sec}$ and extension at $72^{\circ} \mathrm{C}$ for $30 \mathrm{sec}$. The $\mathrm{Cq}$ values were analyzed using the $2^{-\Delta \Delta \mathrm{Cq}}$ method (25). Amplification of the reference endogenous gene GAPDH was used to normalize the data. The primer sequences used for RT-qPCR are listed in Table I.

Cell cycle analysis. 786-O cells with different treatments were fixed with $70 \%$ ethanol for $1 \mathrm{~h}$ at $4^{\circ} \mathrm{C}$ and stained with $50 \mu \mathrm{g} / \mathrm{ml}$ propidium iodide for $30 \mathrm{~min}$ at $4^{\circ} \mathrm{C}$ in the dark. Cell cycle data was acquired using an LSR II flow cytometer (BD Biosciences). Quantification of cells in each phase of the cell cycle was carried out using FlowJo (version 10; FlowJo LLC).

Apoptosis analysis. To quantify apoptosis, annexin $\mathrm{V}$ and propidium iodide staining was carried out, followed by flow cytometry. The 786-O cells with different treatments were collected and washed twice with PBS. Subsequently, Binding Buffer was added to $400 \mu \mathrm{l}$ of cell suspension, stained with $5 \mu \mathrm{l}$ of Annexin V-FITC and propidium iodide (PI) staining solution at room temperature for $15 \mathrm{~min}$ in the dark (all part of a kit; cat. no. C1062S; Beyotime Institute of Biotechnology). Data was acquired using an LSR II flow cytometer (BD Biosciences). Quantification of apoptotic cells was carried out using FlowJo (version 10; FlowJo LLC).

Measurement of mitochondria using MitoTimer. MitoTimer is a novel tool for monitoring real-time mitochondrial aging, turnover and biogenesis, which can be used to evaluate individual mitochondria or mitochondrial populations within a cell (26). MitoTimer encodes a 'timer fluorescent protein' that is targeted to the mitochondrial matrix (26). MitoTimer green fluorescence enters the mitochondrial matrix and matures into a red fluorescent protein $(26,27)$. In this study, MitoTimer plasmid (cat. no. 50547; Biovector NTCC Inc.) was transfected into 293T cells. The plasmid was transfected using Lipofectamine ${ }^{\circledR} 2000$ (Invitrogen; Thermo Fisher Scientific, Inc.) following the manufacturer's recommendations. Cells in the logarithmic growth phase (density, $40-50 \%$ ) were plated in a 6-well plate with $3.5 \mu \mathrm{g}$ plasmid/well and incubated in a $5 \% \mathrm{CO}_{2}$ humidified incubator at $37^{\circ} \mathrm{C}$. Subsequently, $24 \mathrm{~h}$ later production was induced with doxycycline (Dox) $(2 \mu \mathrm{g} / \mathrm{ml})$ at $37^{\circ} \mathrm{C}$ for $24 \mathrm{~h}$. Mitophagy was then observed using a Olympus confocal microscope.

Cell Counting Kit-8 (CCK-8) analysis. The target cells were digested and seeded into a 96 -well plate at a density of $3 \times 10^{3}$ cells. After $24 \mathrm{~h}$ of incubation in an incubator, $10 \mu \mathrm{l}$ CCK-8 reagent was added to each well. After incubation in the incubator for another $2 \mathrm{~h}$, the absorbance was measured at $450 \mathrm{~nm}$ wavelength.

Statistical analysis. Data analysis was performed using Excel (version 16.11.1; Microsoft Corporation) or SPSS for Windows (version 13.0; SPSS, Inc.). Differences between groups were analyzed using Student's unpaired t-tests or one-way ANOVA followed by Tukey's post hoc test. $\mathrm{P}<0.05$ was considered to indicate a statistically significant difference.

\section{Results}

$N R F-1$ has functional binding sites in the promoter region of TFE3. The promoter region online analysis software JASPER 
Table I. Primer sequences used for RT-qPCR.

\begin{tabular}{lll}
\hline Gene name & & \multicolumn{1}{c}{ Primers $\left(5^{\prime}-3^{\prime}\right)$} \\
\hline GAPDH & F: & GGAGCGAGATCCCTCCAAAAT \\
& R: & GGCTGTTGTCATACTTCTCATGG \\
TFE3 & F: & TGTGTACAGTAGTCAAGGCGT \\
& R: & AGTGCCCAGTTCCTTGATCC \\
NRF-1 & F: & GTACAAGAGCATGATCCTGGA \\
& R: & GCTCTTCTGTGCGGACATC \\
mTOR & F: & TCCGAGAGATGAGTCAAGAGG \\
& R: & CACCTTCCACTCCTATGAGGC \\
CCND1 & F: & GCTGCGAAGTGGAAACCATC \\
& R: & CCTCCTTCTGCACACATTTGAA \\
CCND2 & F: & CTGTCTCTGATCCGCAAGCAT \\
& R: & GGTGGGTACATGGCAAACTTAAA \\
AKT & F: & GTCATCGAACGCACCTTCCAT \\
& R: & AGCTTCAGGTACTCAAACTCGT \\
S6 & F: & AGGGTTATGTGGTCCGAATCA \\
& R: & TTGGTCTGTAACAGGAATGCC
\end{tabular}

RT-qPCR, reverse transcription-quantitative; NRF1, nuclear respiratory factor-1; TFE3, IGHM enhancer 3; CCND1, cyclin D1; CCND2, cyclin D2; F, forward; R, reverse.

was used to analyze whether there are NRF-1 binding sites in the promoter region of TFE3. A total of 12 potential binding sites for NRF-1 were identified in the promoter region of TFE3. Primers for the two sites with the highest scores were designed for subsequent experiments (Fig. 1A).

To determine whether the TFE3 promoter interacted with NRF-1, ChIP was performed using genomic DNA and NRF-1-specific antibodies, and qPCR was used to analyzed the target DNA fragments. The ChIP results indicated that NRF-1 could directly bind to the TFE3 promoter region at position 477-487 (Fig. 1B).

The results of the luciferase assay demonstrated luciferase activity of the TFE3 gene promoter in the NRF-1 shRNA group decreased by $\sim 20 \%$ compared with the control group (Fig. 1D). This confirmed that NRF-1 could bind the promoter region of the TFE3 gene, which may have a positive regulatory effect on the transcription of TFE3 gene. Thus, the potential regulatory effect of NRF-1 on TFE3 was further examined. NRF-1 expression was silenced using shRNA, and the mRNA and protein expression levels of TFE3 were measured. The results indicated that NRF-1 silencing reduced the expression of TFE3 compared with NRF-1 NC (Fig. 2A-C). Moreover, the mRNA and protein expression levels of TFE3 were measured following NRF-1 overexpression. The mRNA and protein levels of TFE3 were upregulated following NRF-1 overexpression, which was consistent with the aforementioned results (Fig. 2D-F).

Function of NRF-1 in kidney cancer cells. TFE3 have also been implicated in mTOR signaling, a major regulator of protein synthesis contributing to the growth of several tumor types, including RCC (28). NRF-1 expression was silenced in 786-O cells using shRNA and the expression of mTOR-related indicators (mTOR, AKT/p-AKT and $\mathrm{S} 6 / \mathrm{p}-\mathrm{S} 6)$ was measured. The protein levels of $\mathrm{p}-\mathrm{AKT}$ and p-S6 were decreased compared with the control group and the results showed that the expression levels of components of the mTOR pathway were downregulated following NRF-1 shRNA transfection (Fig. 3A-C). In order to further verify that NRF-1 can regulate the mTOR pathway through TFE3, the TFE3 protein was overexpressed in NRF-1 shRNA 786-O cells. The overexpression plasmid of TFE3 was verified using western blot analysis (Fig. S1). The results showed that the overexpression of the TFE3 protein together with NRF-1 silencing restored the expression mTOR pathway-associated proteins compared with NRF-1 or TFE3 silencing alone (Fig. 3D and E).

In order to detect the effect of NRF-1 on tumor cell apoptosis and proliferation, flow cytometry was used following NRF-1 silencing using shRNA. The results demonstrated that NRF-1 silencing promoted the apoptosis of 786-O cells (Fig. 4A and B). Compared with the control group, NRF-1 shRNA 786-O cells showed an increase in the fraction of cells in the $G_{1}$ phase and a decrease in the $G_{2}$ phase of the cell cycle. These results indicated that NRF-1 silencing resulted in $\mathrm{G}_{1}$ cycle arrest (Fig. 4C and D).

Further experiments showed that TFE3 and NRF-1 silencing promote cell apoptosis to varying degrees. Indeed, following simultaneous NRF-1 silencing and TFE3 overexpression, the apoptosis of 786-O cells was significantly reduced compared with all other groups (Fig. 5A and C). These results indicated that TFE3 played a key role in tumor cell apoptosis. NRF-1 can participate in the regulation of cell apoptosis by directly regulating the expression of TFE3 protein.

NRF-1 and TFE3 silencing alone resulted in $\mathrm{G}_{1}$-phase cell cycle arrest in 786-O cells. By contrast, following NRF-1 silencing and TFE3 overexpression, cell cycle arrest in $\mathrm{G}_{1}$ phase was suppressed, restoring the fraction of cells to levels similar to those of the control group (Fig. 5B and D).

In order to detect changes in cell proliferation, CCK-8 was used. Compared with the control group, NRF-1 and TFE3 silencing alone inhibited cell proliferation. However, following TFE3 overexpression in NRF-1 shRNA cells, proliferation increased compared with NRF-1 and TFE3 silencing alone (Fig. 5E).

Effect of NRF-1 expression on mitochondria production of tumor cells. To determine how changes in NRF expression might affect mitochondria, the MitoTimer plasmid to detect changes in mitochondrial formation in cells. Under the same shooting conditions, significantly reduced fluorescence signals were observed in the NRF-1 shRNA group, and down-regulation of mitochondrial generation was observed. The results showed that the fluorescence of cells in the NRF-1 shRNA group showed a significant decrease compared with the NC (Fig. 6). Thus, the mitochondrial generation rate was slowed down following knockdown of NRF-1.

\section{Discussion}

In the present study, the expression of the TFE3 gene was directly regulated by NRF-1, a crucial transcription factor involved in 
A

$$
\text { Possible NRF-1 binding sites }
$$

$\begin{array}{lr}\text { Position } & \text { Sequence, } 5^{\prime} \text { to } 3^{\prime} \\ 477 / 487 & \text { GCGCCTGCGCA } \\ 941 / 951 & \text { CCGCCCGCGCA }\end{array}$

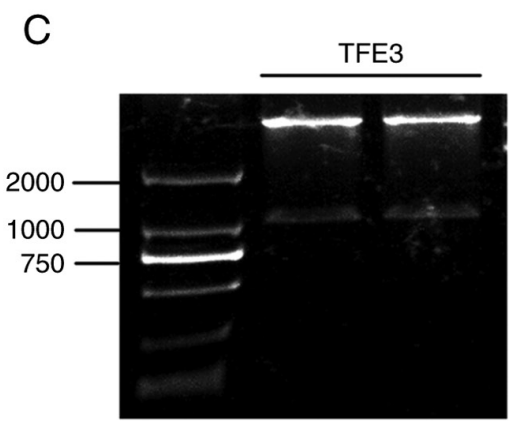

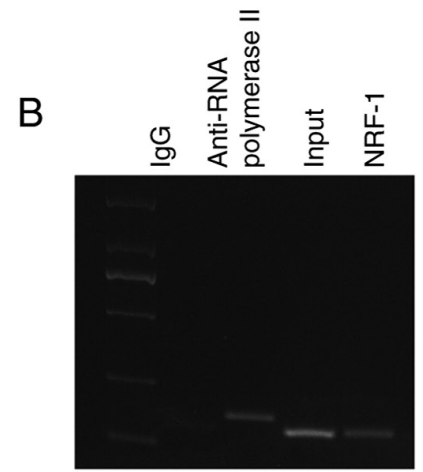

D

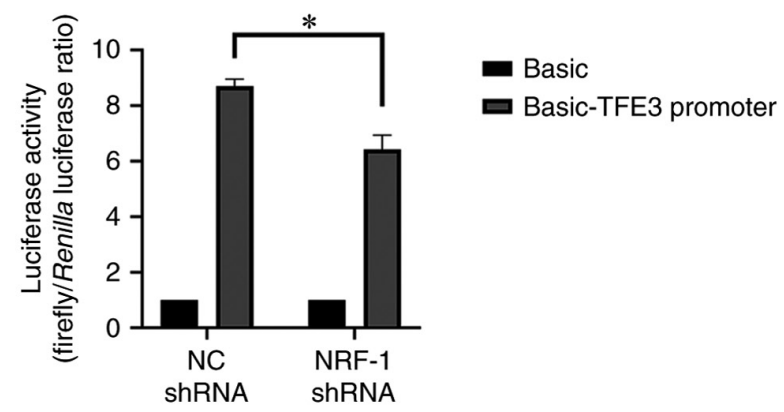

Figure 1. NRF-1 has functional binding sites in the promoter regions of TFE3. (A) Potential binding sites of NRF-1 in the promoter region of the TFE3 gene. (B) NRF-1 has a binding site to the promoter region of TFE3 at position 477-487 bp. (C) Construction of luciferase reporter gene in promoter region of TFE3 gene and detection of luciferase activity in promoter region. (D) Luciferase activity was measured as the ratio of the firefly to Renilla fluorescence signal and normalized to the empty plasmid. Data are presented as the mean \pm SEM. $n=3$. $P<0.05$. NRF-1, nuclear respiratory factor-1; TFE3, transcription factor binding to IGHM enhancer 3, NC, negative control; Ig, immunoglobulin; shRNA, short hairpin RNA.

A

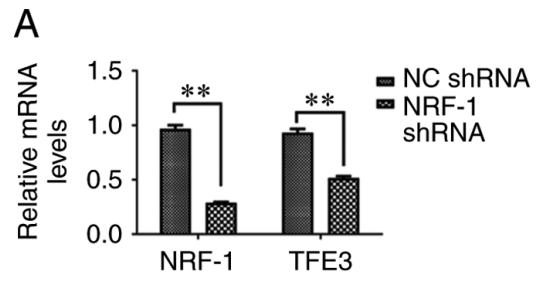

$\mathrm{D}$

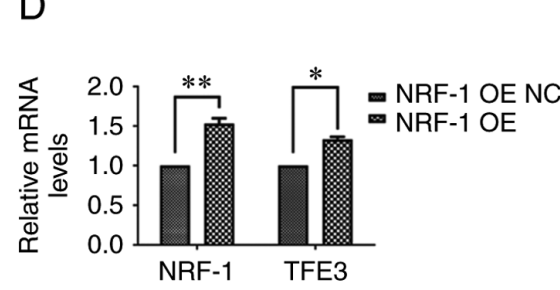

B

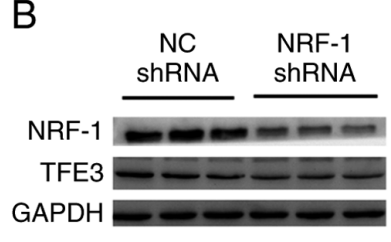

$E$

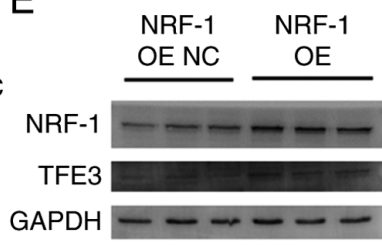

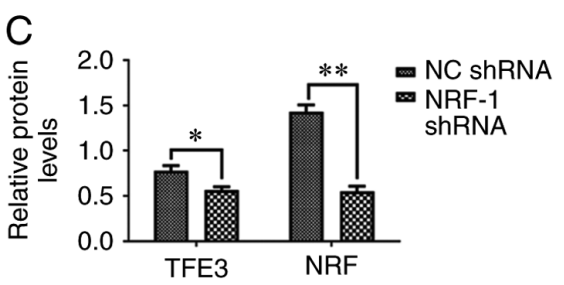

$\mathrm{F}$

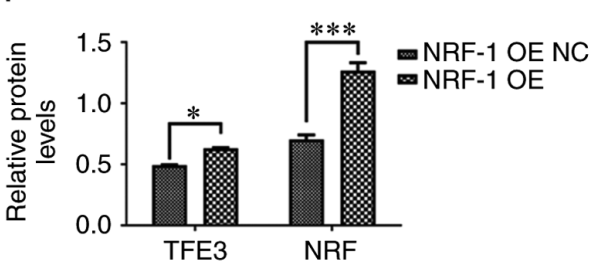

Figure 2. Positive regulation of TFE3 by NRF-1. (A) NRF-1 and TFE3 mRNA expression levels decreased following NRF-1 knockdown in 786-O cells. (B and C) NRF-1 and TFE3 protein expression levels decreased following NRF-1 knockdown in 786-O cells. (D) NRF-1 and TFE3 mRNA expression levels following NRF-1 OE in 786-O cells. (E and F) NRF-1 and TFE3 protein expression levels increased following NRF-1 OE in 786-O cells. Data are presented as the mean \pm SEM. $n=3 .{ }^{*} \mathrm{P}<0.05,{ }^{* *} \mathrm{P}<0.01,{ }^{* * *} \mathrm{P}<0.005$. NRF-1, nuclear respiratory factor-1; TFE3, transcription factor binding to IGHM enhancer 3; NC, negative control; shRNA, short hairpin RNA; OE, overexpression.

oxidative phosphorylation and mitochondrial biogenesis (19). The role of TFE3 and its fusion gene in renal cancer has not been fully elucidated since its first discovery $(9,10)$. The occurrence and progression of tumors are closely related to energy metabolism and NRF-1 coordinates synaptic activity and energy metabolism $(22,29)$. Previous reports have indicated that TFE3 may be one of the downstream target genes of NRF-1 (30).
Therefore, the aim of the present study was to confirm the regulatory relationship between NRF-1 and TFE3, in order to explore the role of NRF-1/TFE3 in the process of tumorigenesis.

A previous study on NRF-1 in the literature have focused on mitochondria and energy metabolism (31). Autophagy, as well as tumor-related autophagy and even cellular immunity (32-35). However, there is less research on the interaction between NRF-1 
A
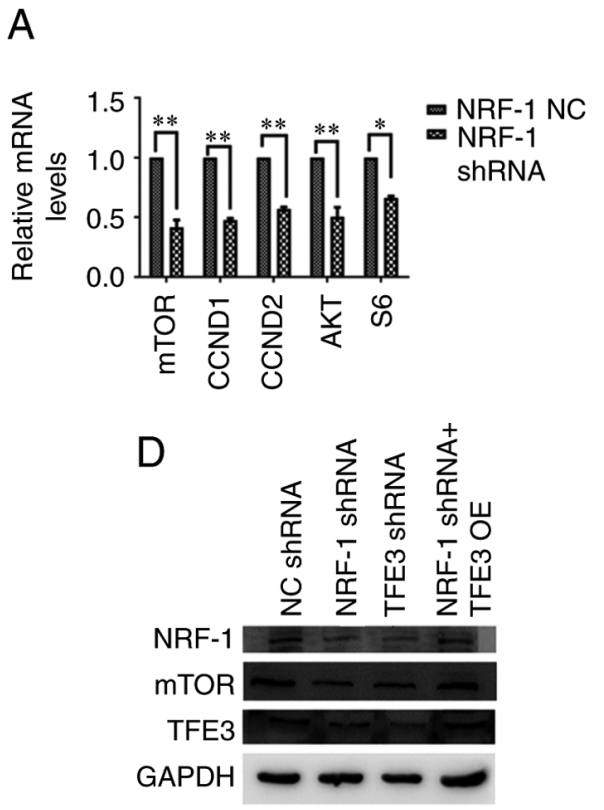

B

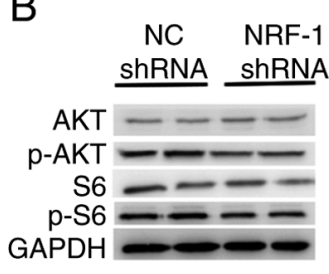

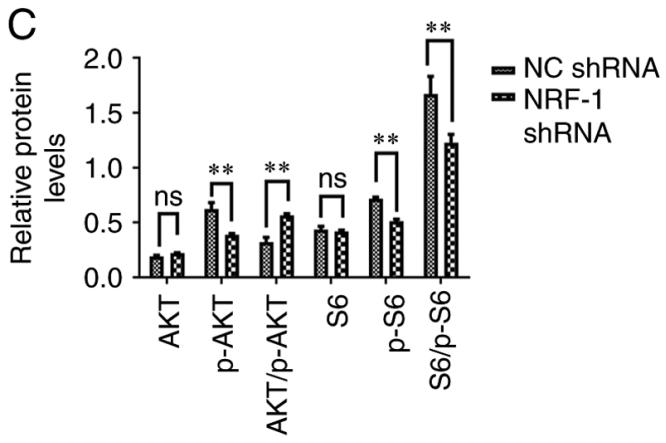

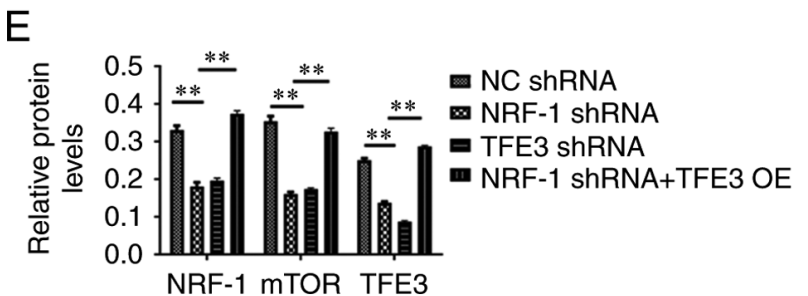

Figure 3. NRF-1 regulates the mTOR pathway. (A) mRNA expression levels of mTOR, CCND1, CCND2, AKT and S6 following shRNA-mediated NRF-1 knockdown. (B and C) AKT and S6 phosphorylation levels following shRNA-mediated NRF-1 knockdown. (D and E) NRF-1, mTOR and TFE3 protein expression levels following NRF-1 knockdown with or without TFE3 OE. Data are presented as the mean \pm SEM. $n=3$. ${ }^{*} \mathrm{P}<0.05,{ }^{* *} \mathrm{P}<0.01$. CCND, cyclin $\mathrm{D}$; S6, S6 ribosomal protein; NRF-1, nuclear respiratory factor-1; TFE3, transcription factor binding to IGHM enhancer 3; NC, negative control; shRNA, short hairpin RNA; OE, overexpression; ns, not significant; p-, phosphorylated.

A

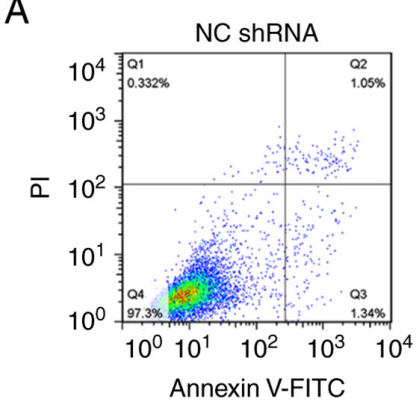

C

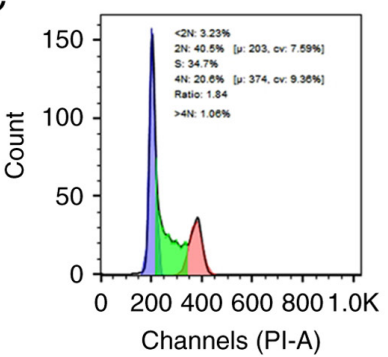

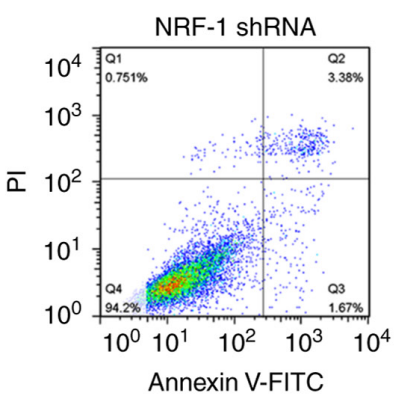

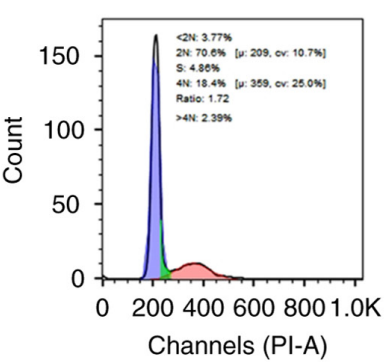

B
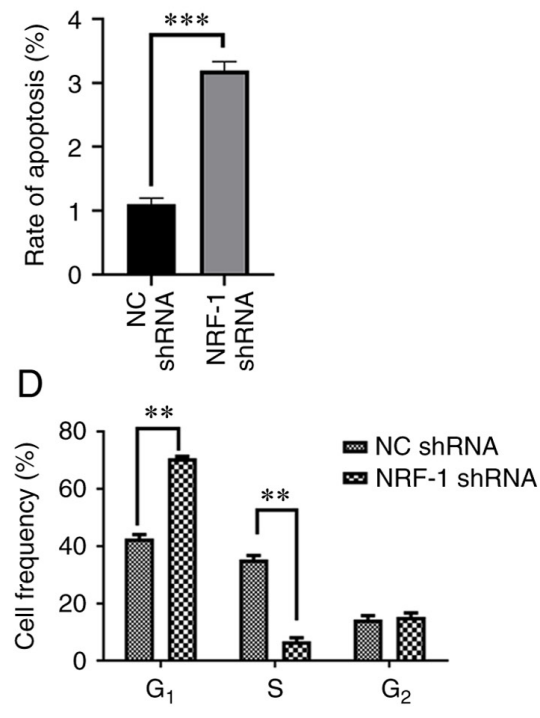

Figure 4. Increased levels of apoptosis and cell cycle arrest. (A and B) Apoptosis rates increased following NRF-1 knockdown in 786-O cells. (C and D) NRF-1 knockdown resulted in G1-phase cell cycle arrest in 786-O cells. Data are presented as the mean $\pm \mathrm{SEM} . \mathrm{n}=3$. ${ }^{* *} \mathrm{P}<0.01,{ }^{* * * *} \mathrm{P}<0.005$. NRF-1, nuclear respiratory factor-1; NC, negative control; shRNA, short hairpin RNA; PI, propidium iodide.

and TFE3. In the present study, NRF-1 directly regulated the expression of the TFE3 protein. The present findings confirmed that NRF-1 could functionally bind to the TFE3 promoter region, thereby regulating the expression of the TFE 3 protein. The $\mathrm{mTOR}$ signaling pathway is activated in a variety of cancer types (36). It regulates the metabolism of amino acids, glucose, nucleotides, fatty acids and lipids by changing the expression and/or activity of several key metabolic enzymes and participates in the control of cell growth and metabolism (36). Moreover, studies have shown that metabolic changes, such as increased glucose or amino acid intake, can affect mTOR signaling $(37,38)$. The present results indicated that following NRF-1 knockdown, the expression levels of TFE3 and components of the mTOR signaling pathway were also downregulated. The rate of apoptosis also increased, while cell proliferation was suppressed. These results indicated that NRF-1 regulated the mTOR pathway through TFE3. 
A

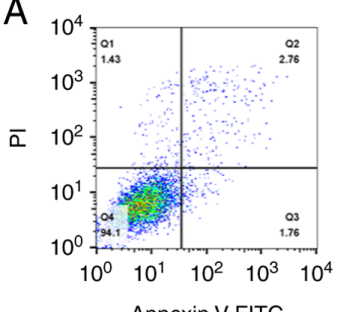

Annexin V-FITC

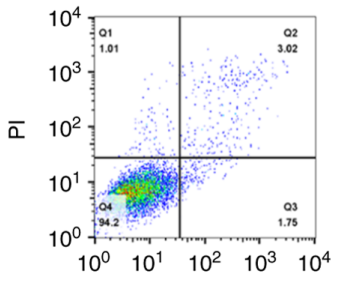

Annexin V-FITC

C

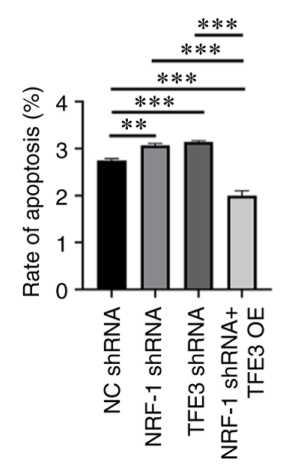

B
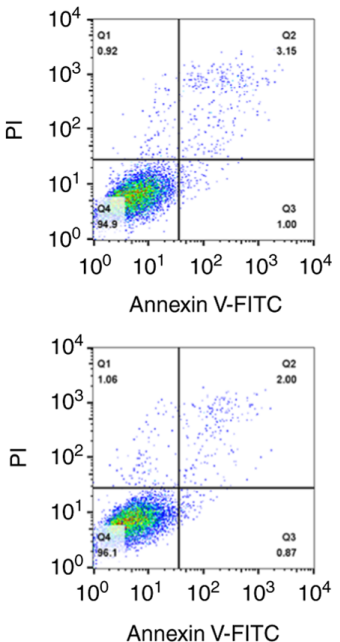

D

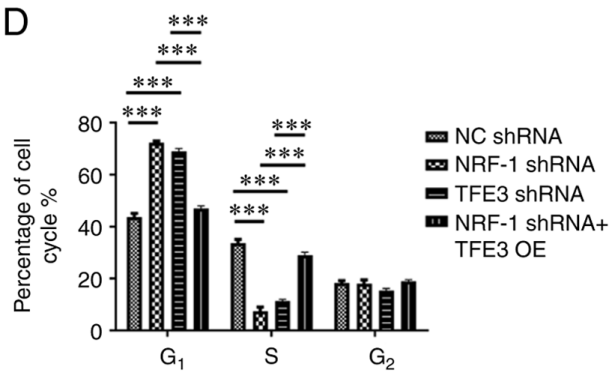

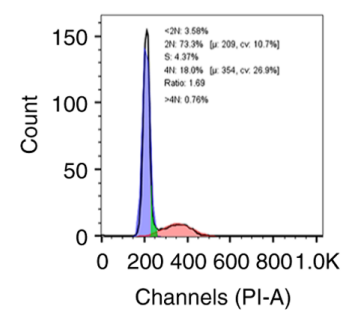

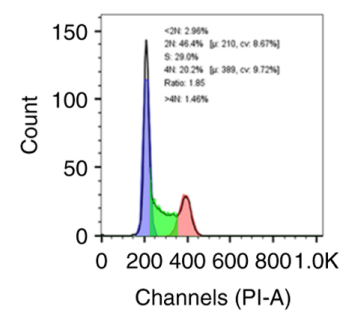

E

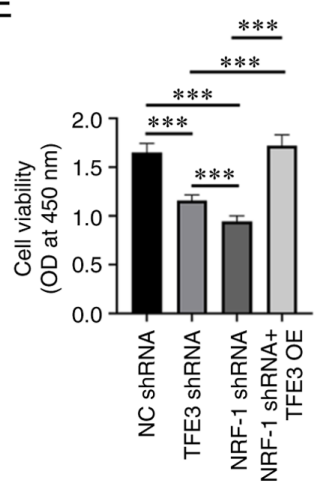

Figure 5. NRF-1/TFE3 promotes tumor cell proliferation and inhibits tumor cell apoptosis. (A and C) Apoptosis rates increased following NRF-1 or TFE3 knockdown in 786-O cells, but were reduced following co-transfection with NRF-1 shRNA and TFE3 OE. (B and D) NRF-1 or TFE3 knockdown resulted in G1-phase cell cycle arrest in 786-O cells; whereas TFE3 OE reversed this effect. (E) Cell viability in transfected cells. Data are presented as the mean \pm SEM. $\mathrm{n}=3 .{ }^{* *} \mathrm{P}<0.01,{ }^{* * *} \mathrm{P}<0.005$. NRF-1, nuclear respiratory factor-1; TFE3, transcription factor binding to IGHM enhancer 3; NC, negative control; shRNA, short hairpin RNA; OE, overexpression.
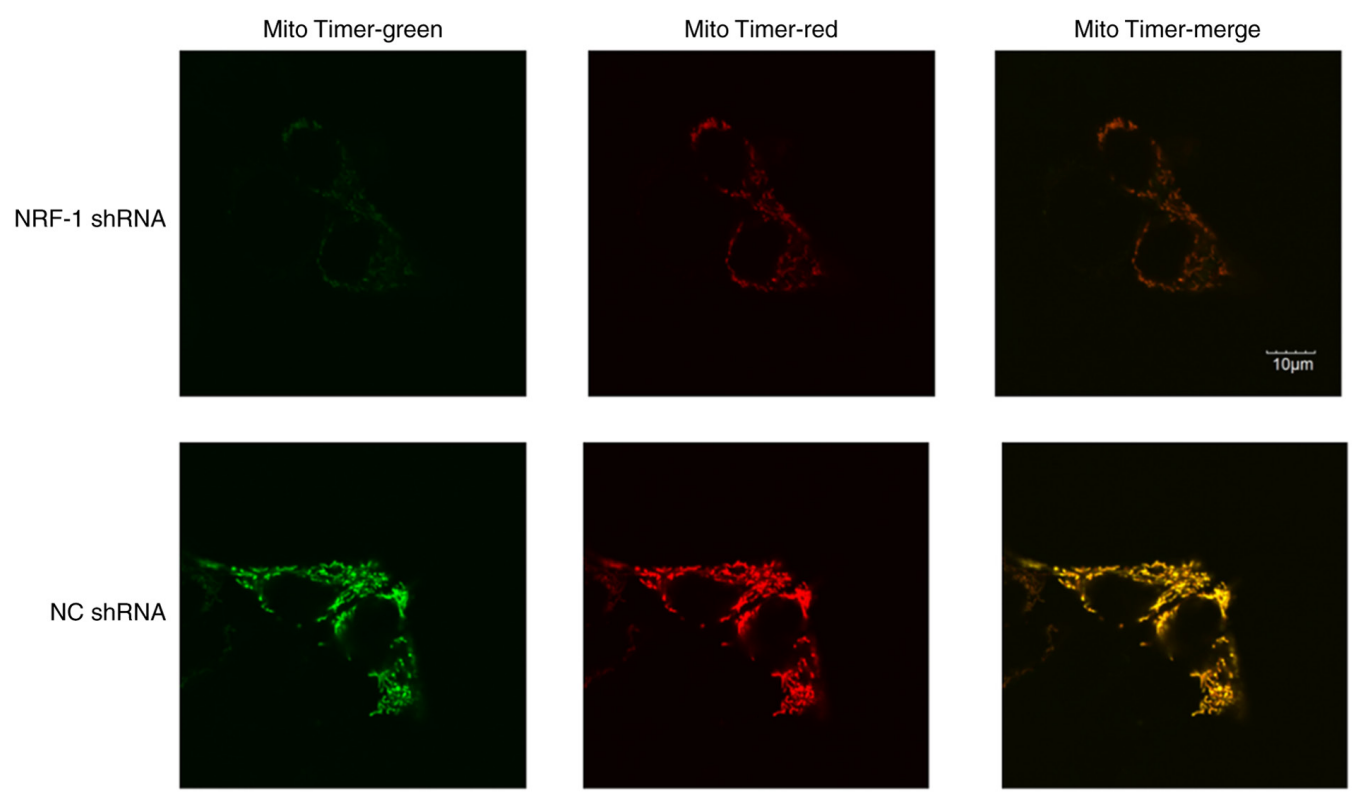

Figure 6. NRF-1 downregulation reduces mitochondrial production . 293T cells were co-transfected with the MitoTimer plasmid together with NRF-1 NC or NRF-1 shRNA. After $24 \mathrm{~h}$, DOX was added to induce the expression of MitoTimer plasmid for $24 \mathrm{~h}$. Mito-timer green fluorescent protein entered the mitochondrial matrix and matured into a red fluorescent protein. The amount of green fluorescence that entered the mitochondrial matrix and matured into red fluorescent protein depends on the rate of mitochondrial biosynthesis. NRF-1, nuclear respiratory factor-1; NC, negative control; shRNA, short hairpin RNA; DOX, doxycycline. 
A variety of signaling pathways are mediated by TFE3, the dysregulation of which might contribute to renal carcinogenesis (39). A previous study have suggested that TFE3 can be a target of the mTOR signaling pathway and is regulated by mTOR signaling (40). However, a previous study involving CHIP-Seq detection of the SFPQ-TFE3 fusion gene have identified other target genes that were related to PI3K/AKT/mTOR, including PI3KCA, TSC1, AKT3, PTEN, 14-3-3, ITGB1, IGFR1 and IRS-1 genes (41). This may also explain why, in addition to being regulated by mTOR, TFE3 can also positively regulate the expression of mTOR. In the present study, simultaneous overexpression of TFE3 and NRF-1 silencing restored the downregulated expression of molecules involved in the mTOR signaling pathway, inhibited apoptosis, and increased proliferation. These results indicated that the TFE3 protein itself could also play a positive regulatory role on the mTOR signaling pathway. The TFE3 protein is directly related to the apoptosis and growth of cells. This is also consistent with a previous study (42). There may be a circular relationship between TFE3 and mTOR. Moreover, the present study also confirmed the direct regulation of NRF-1 on the TFE3 gene. Further experiments are needed to determine whether there is a circular relationship between TFE3 and NRF-1.

NRF-1 itself is involved in the production of mitochondria and the regulation of cell energy metabolism under various conditions (43), and there are few studies on the role of NRF-1 in renal cell carcinoma (23). For tumor cells, metabolic remodeling is an important feature (44). This allows tumor cells to adapt to various environments and survive under the body's immune system (22). TFE3 plays a key role in certain renal cell carcinoma types, and NRF-1, as its upstream regulatory gene, is highly related to its expression level (23). These findings may provide insight into TFE3-positive renal cancer treatment and suggest that NRF-1 may become a new target for the treatment of this condition.

Given the important role of TFE3 in tumors, particularly tRCC, TFE3 may be an important target for researching tumor treatment measures. As a direct upstream regulatory gene of TFE3, NRF-1 may also play a key role in this process. This study showed that NRF-1 directly regulates TFE3 and may influence downstream tumorigenesis and progression through TFE3. Therefore, understanding the specific regulatory effect of NRF-1 on TFE3 may help discover new therapeutic targets for renal tumors.

\section{Acknowledgements}

Not applicable.

\section{Funding}

This work was supported by The National Natural Science Foundation of China (grant no. 81572512) and The Beijing Ronghe Medical Development Foundation. The funders had no role in study design, data collection and analysis, decision to publish, or preparation of the manuscript.

\section{Availability of data and materials}

The datasets used and/or analyzed during the current study are available from the corresponding author on reasonable request.

\section{Authors' contributions}

WDG, CNZ and HQG conceived and designed the experiments. WYZ, XD, BW and NL performed the experiments and analyzed data. WYZ and XD wrote the manuscript. WDG and WYZ confirmed the authenticity of all the raw data. All authors read and approved the final manuscript.

\section{Ethics approval and consent to participate}

Not applicable.

\section{Patient consent for publication}

Not applicable.

\section{Competing interests}

The authors declare that they have no competing interests.

\section{References}

1. Betschinger J, Nichols J, Dietmann S, Corrin PD, Paddison PJ and Smith A: Exit from pluripotency is gated by intracellular redistribution of the bHLH transcription factor Tfe3. Cell 153: 335-347, 2013.

2. Fisher DE, Carr CS, Parent LA and Sharp PA: TFEB has DNA-binding and oligomerization properties of a unique helix-loop-helix/leucine-zipper family. Genes Dev 5: 2342-2352, 1991.

3. Hemesath TJ, Steingrímsson E, Mcgill G, Hansen MJ, Vaught J, Hodgkinson CA, Arnheiter H, Copeland NG, Jenkins NA and Fisher DE: microphthalmia, a critical factor in melanocyte development, defines a discrete transcription factor family. Genes Dev 8: 2770-2780, 1994.

4. Muhle-Goll C, Gibson T, Schuck P, Schubert D, Nalis D, Nilges M and Pastore A: The dimerization stability of the HLH-LZ transcription protein family is modulated by the leucine zippers: A CD and NMR study of TFEB and c-Myc. Biochemistry 33: 11296-11306, 1994.

5. Vivian P, Ogmundsdóttir MH, Bergsteinsdóttir K, Schepsky A, Phung B, Deineko V, Milewski M, Steingrímsson E and Wilmanns M: Restricted leucine zipper dimerization and specificity of DNA recognition of the melanocyte master regulator MITF. Genes Dev 26: 2647-2658, 2012.

6. Raben $\mathrm{N}$ and Puertollano R: TFEB and TFE3: Linking lysosomes to cellular adaptation to stress. Annu Rev Cell Dev Biol 32: 255-278, 2016.

7. Xiong L, Chen X, Liu N, Wang Z, Miao B, Gan W, Li D and Guo H: PRCC-TFE3 dual-fusion FISH assay: A new method for identifying PRCC-TFE3 renal cell carcinoma in paraffin-embedded tissue. PLoS One 12: e0185337, 2017.

8. Magers MJ, Udager AM and Mehra R: MiT family translocation-associated renal cell carcinoma: A contemporary update with emphasis on morphologic, immunophenotypic, and molecular mimics. Arch Pathol Lab Med 139: 1224-1233, 2015.

9. Martina JA, Diab HI, Brady OA and Puertollano R: TFEB and TFE3 are novel components of the integrated stress response. EMBO J 35: 479-495, 2016.

10. Pastore N, Vainshtein A, Klisch TJ,Armani A, Huynh T, Herz NJ, Polishchuk EV, Sandri $M$ and Ballabio A: TFE3 regulates whole-body energy metabolism in cooperation with TFEB. EMBO Mol Med 9: 605-621, 2017.

11. Martina JA, Diab HI, Lishu L, Jeong-A L, Patange S, Raben N and Puertollano R: The nutrient-responsive transcription factor TFE3 promotes autophagy, lysosomal biogenesis, and clearance of cellular debris. Sci Signal 7: ra9, 2014.

12. Ploper D, Taelman VF, Robert L, Perez BS, Titz B, Chen HW, Graeber TG, von Euw E, Ribas A and De Robertis EM: MITF drives endolysosomal biogenesis and potentiates Wnt signaling in melanoma cells. Proc Natl Acad Sci USA 112: E420-E429, 2015. 
13. Iwasaki H, Naka A, Iida KT, Nakagawa Y, Shimano H, Matsuzaka T, Ishii KA, Kobayashi K, Takahashi A, Yatoh S, et al: TFE3 regulates muscle metabolic gene expression, increases glycogen stores, and enhances insulin sensitivity in mice. Am J Physiol Endocrinol Metab 302: E896-E902, 2012.

14. Nakagawa Y, Shimano H, Yoshikawa T, Ide T, Tamura M, Furusawa M, Yamamoto T, Inoue N, Matsuzaka T, Takahashi A, et al: TFE3 transcriptionally activates hepatic IRS-2, participates in insulin signaling and ameliorates diabetes. Nat Med 12: 107-113, 2006.

15. Nijman SMB, Hijmans EM, Messaoudi SE, van Dongen MMW, Sardet $C$ and Bernards R: A functional genetic screen identifies TFE3 as a gene that confers resistance to the anti-proliferative effects of the retinoblastoma protein and transforming growth factor-beta. J Biol Chem 281: 21582-21587, 2006.

16. Muller-Hocker J, Babaryka G, Schmid I and Jung A: Overexpression of cyclin D1, D3, and p21 in an infantile renal carcinoma with Xp11.2 TFE3-gene fusion. Pathol Res Pract 204 589-597, 2008

17. Scarpulla RC: Nuclear control of respiratory gene expression in mammalian cells. J Cell Biochem 97: 673-683, 2010.

18. Scarpulla RC: Nuclear control of respiratory chain expression by nuclear respiratory factors and PGC-1-related coactivator. Ann N Y Acad Sci 1147: 321-334, 2010

19. Virbasius CA, Virbasius JV and Scarpulla RC: NRF-1, an activator involved in nuclear-mitochondrial interactions, utilizes a new DNA-binding domain conserved in a family of developmental regulators. Genes Dev 7: 2431, 1993.

20. Tang M, Yang Y, Yu J, Qiu J, Chen P, Wu Y, Wang Q, Xu Z, Ge J, $\mathrm{Yu} \mathrm{K}$ and Zhuang J: Tetramethylpyrazine in a murine alkali-burn model blocks NFKB/NRF-1/CXCR4-signaling-induced corneal neovascularization. Invest Ophthalmol Vis Sci 59: 2133-2141, 2018.

21. Satoh J, Kawana N and Yamamoto Y: Pathway analysis of chip-seq-based NRF1 target genes suggests a logical hypothesis of their involvement in the pathogenesis of neurodegenerative diseases. Gene Regul Syst Bio 7: 139-152, 2013.

22. Kimmelman AC and White E: Autophagy and tumor metabolism. Cell Metab 25: 1037-1043, 2017.

23. Alam C, Hoque MT, Sangha V and Bendayan R: Nuclear respiratory factor 1 (NRF-1) upregulates the expression and function of reduced folate carrier (RFC) at the blood-brain barrier. FASEB J 34: 10516-10530, 2020.

24. Solecki D, Bernhardt G, Lipp M and Wimmer E: Identification of a nuclear respiratory Factor-1 binding site within the core promoter of the human polio virus receptor/CD155 Gene. J Biol Chem 275: 12453-12462, 2000.

25. Livak KJ and Schmittgen TD: Analysis of relative gene expression data using real-time quantitative PCR and the 2(-Delta Delta C(T)) method. Methods 25: 402-408, 2001

26. Hernandez G, Thornton C, Stotland A, Lui D, Sin J, Ramil J, Magee N, Andres A, Quarato G, Carreira RS, et al: MitoTimer: A novel tool for monitoring mitochondrial turnover. Autophagy 9 : $1852-1861,2013$

27. Williams JA, Zhao K, Jin S and Ding WX: New methods for monitoring mitochondrial biogenesis and mitophagy in vitro and in vivo. Exp Biol Med (Maywood) 242: 781-787, 2017.

28. Motzer RJ, Escudier B, Oudard S, Hutson TE, Porta C, Bracarda S, Grünwald V, Thompson JA, Figlin RA, Hollaender N, et al: Efficacy of everolimus in advanced renal cell carcinoma: A double-blind, randomised, placebo-controlled phase III trial. Lancet 372: 449-456, 2008

29. Scarpulla RC: Metabolic control of mitochondrial biogenesis through the PGC-1 family regulatory network. Biochim Biophys Acta 1813: 1269-1278, 2011.
30. Jun-Ichi S, Natsuki K and Yoji Y: Pathway analysis of ChIP-Seq-Based NRF1 target genes suggests a logical hypothesis of their involvement in the pathogenesis of neurodegenerative diseases. Gene Regul Syst Bio 7: 139-152, 2013.

31. Evans MJ and Scarpulla RC: NRF-1: A trans-activator of nuclear-encoded respiratory genes in animal cells. Genes Dev 4: 1023-1034, 1990.

32. Taniguchi M, Nadanaka S, Tanakura S, Sawaguchi S, Midori S, Kawai Y, Yamaguchi S, Shimada Y, Nakamura Y, Matsumura Y, et al: TFE3 is a bHLH-ZIP-type transcription factor that regulates the mammalian Golgi stress response. Cell Struct Funct 40: 13-30, 2015.

33. Zanocco-Marani T, Vignudelli T, Parenti S, Gemelli C, Condorelli F, Martello A, Selmi T, Grande A and Ferrari S: TFE3 transcription factor regulates the expression of MAFB during macrophage differentiation. Exp Cell Res 315: 1798-1808, 2009.

34. Zanocco-Marani T, Vignudelli T, Gemelli C, Pirondi S, Testa A, Montanari M, Parenti S, Tenedini E, Grande A and Ferrari S: Tfe 3 expression is closely associated to macrophage terminal differentiation of human hematopoietic myeloid precursors. Exp Cell Res 312: 4079-4089, 2006.

35. Shi CS, Shenderov K, Huang NN, Kabat J, Abu-Asab M, Fitzgerald KA, Sher A and Kehrl JH: Activation of autophagy by inflammatory signals limits IL- $1 \beta$ production by targeting ubiquitinated inflammasomes for destruction. Nat Immunol 13: 255-263, 2012.

36. Murugan AK: mTOR: Role in cancer, metastasis and drug resistance. Semin Cancer Biol 59: 92-111, 2019.

37. Saxton RA and Sabatini DM: mTOR signaling in growth, metabolism, and disease. Cell 168: 960-976, 2017.

38. Zhang Y, Kwok-Shing Ng P, Kucherlapati M, Chen F, Liu Y, Tsang YH, de Velasco G, Jeong KJ, Akbani R, Hadjipanayis A, et al: A Pan-cancer proteogenomic Atlas of $\mathrm{PI} 3 \mathrm{~K} / \mathrm{AKT} / \mathrm{mTOR}$ pathway alterations. Cancer Cell 31: 820-832. e3, 2017.

39. Argani P, Hicks J, De Marzo AM, Albadine R, Illei PB, Ladanyi M, Reuter VE and Netto GJ: Xp11 translocation renal cell carcinoma (RCC): Extended immunohistochemical profile emphasizing novel RCC markers. Am J Surg Pathol 34: 1295-1303, 2010.

40. Li J, Wada S, Weaver LK, Biswas C, Behrens EM and Arany Z: Myeloid Folliculin balances mTOR activation to maintain innate immunity homeostasis. JCI Insight 5: e126939, 2019.

41. Damayanti NP, Budka JA, Khella HWZ, Ferris MW, $\mathrm{Ku}$ SY, Kauffman E, Wood AC, Ahmed K, Chintala VN, Adelaiye-Ogala $\mathrm{R}$, et al: Therapeutic targeting of TFE3/IRS-1/PI3K/mTOR axis in translocation renal cell carcinoma. Clin Cancer Res 24: 5977-5989, 2018.

42. Kauffman EC, Ricketts CJ, Rais-Bahrami S,Yang Y, Merino MJ, Bottaro DP, Srinivasan R and Linehan WM: Molecular genetics and cellular features of TFE3 and TFEB fusion kidney cancers. Nat Rev Urol 11: 465-475, 2014.

43. Wang D, Zhang J, Lu Y, Luo Q and Zhu L: Nuclear respiratory factor-1 (NRF-1) regulated hypoxia-inducible factor-1 $\alpha$ (HIF-1 $\alpha$ ) under hypoxia in HEK293T. IUBMB Life 68: 748-755, 2016.

44. Li X, Wenes M, Romero P, Huang CC, Fendt SM and Ho PC: Navigating metabolic pathways to enhance antitumour immunity and immunotherapy. Nat Rev Clin Oncol 16: 425-441, 2019.

This work is licensed under a Creative Commons Attribution-NonCommercial-NoDerivatives 4.0 International (CC BY-NC-ND 4.0) License. 